\title{
Modelling of the elementary surface processes occurring in the course of heterogeneous catalytic reactions
}

\author{
M.F.Holovko, E.V.Vakarin \\ Institute for Condensed Matter Physics \\ of the National Academy of Sciences of Ukraine, \\ 1 Svientsitskii Str., 79011 Lviv, Ukraine
}

Received April 18, 2000

\begin{abstract}
A short review of theoretical results on the Langmuir-Hinschelwood and Eley-Rideal reaction schemes is presented. It is shown that the rates of elementary processes at the surface are coupled through the bulk properties of adsorbing species. This concerns the dependence of the chemical potential on the association-dissociation balance and on the adsorbate density near the surface. Adsorption is shown to be sensitive to a mechanism of dimer adsorption (associative or dissociative). Dissociative adsorption gives a nonmonotonic behaviour of the coverage with the surface activity. Simultaneously, the dissociation degree increases with increasing adsorption. The associative adsorption implies the existence of a low-density critical point around which the adsorption isotherms are discontinuous. This causes an abnormal low surface diffusion at low densities. Implementation of these results to kinetic properties are briefly discussed.
\end{abstract}

Key words: heterogeneous catalysis, two-dimensional lattice gas, dissociative and associative adsorption, surface diffusion

PACS: $68.10 . J y, 82.65 . J V$

\section{Introduction}

It is a real pleasure for us to contribute this article to the special issue dedicated to I.R.Yukhnovskii, the discussions with whom have stimulated our interest to the problems of heterogeneous catalysis.

Heterogeneous catalysis is a multistep process involving a dissociative adsorption of reactants followed by the reaction and desorption of the products [1]. Despite a considerable progress in the field, our comprehension of the basic processes involved into catalytic reactions is still incomplete. The main difficulty arises from a very delicate interplay of the metal and the gas parts in accelerating any specific reaction $[2]$. 
Calculation of the rate of elementary surface processes (adsorption, desorption, diffusion, reaction, etc) is a difficult problem, involving both the dynamics and the statistics of the adsorbed particles and surface atoms. As a first approximation, the dynamical and statistical parts of the problem are assumed to be decoupled. That is, the coverage dependence of the process rate is determined by the statistics of adsorbate and metal particles. In other words, the coverage dependence is then calculated through a solution of an equilibrium problem. From this point of view, it is important to make a link between a rapid progress in the field of equilibrium properties and our relatively modest knowledge of kinetic aspects.

In this respect many important questions remain to be answered [3]. For instance, does the reaction occur between two chemisorbed species or between a chemisorbed particle and a gas particle? Considering as an example the oxidation of $\mathrm{CO}$ on $\mathrm{Pt}(111)$, we have two alternative reaction schemes:

Langmuir-Hinschelwood (LH) mechanism

$$
\mathrm{CO} \rightarrow \mathrm{CO}(\text { ads }), \quad \mathrm{O}_{2} \rightarrow 2 \mathrm{O}(\text { ads }), \quad \mathrm{CO}(\text { ads })+\mathrm{O}(\text { ads }) \rightarrow \mathrm{CO}_{2}
$$

and Eley-Rideal (ER) mechanism

$$
\mathrm{O}_{2} \rightarrow 2 \mathrm{O}(\mathrm{ads}), \quad \mathrm{O}(\mathrm{ads})+\mathrm{CO} \rightarrow \mathrm{CO}_{2} .
$$

Although it remains a fact that traditional kinetic measurements do not clearly distinguish between the two, the LH mechanism is commonly discussed as a leading process for this reaction. A kinetic model based on LH scenario, was proposed [4] by Ziff, Gulari and Barshad (ZGB). Nevertheless the ER scenario is not negligible in application to other processes [5].

Notice that (1) or (2) do not follow from any microscopic consideration. These are only examples of semiempirical rules which are widely used as extreme cases in describing catalytic reactions. One can easily imagine a process which is a superposition of these two. Therefore, a sequence of elementary steps remains to be investigated. This implies that a discussion starting from a microscopic model would be much useful for a selfconsistent description.

Another shortcoming of standard approaches is a purely two-dimensional description. Properties of the fluid bulk are represented by an external parameter (impingement rates, or partial pressures). Some of them are indeed marginal for dilute gases which exist in a single phase. But in general this is not true, especially for dense adsorbate systems, which may exhibit peculiar features (liquid-gas coexistence, clustering or phase separation). Thus, a microscopic consideration would allow us to make a connection between the kinetic coefficients and properties of the gas phase. This connection may have important consequences because, for dense systems, an entropic attractive potential appears even if the bare interaction is repulsive. The attraction causes the formation of adsorbed domains and may significantly change [6] the kinetic coefficients.

Dissociation of diatomic molecules prior to their adsorption is usually taken into account implicitly. That is, the dissociation is only declared but is not described assuming that the process takes place near the surface but not at the surface. This 
is another drawback of a two-dimensional description. Nevertheless, the coverage should be sensitive to the dissociation mechanism. Otherwise, adsorption molecules (e.g. $\mathrm{O}_{2}$ ) or monomeric species (2O atoms) would be almost equally probable.

The aim of this paper is to address some of these points. We show that the two-dimensional Lattice Gas (LG) Hamiltonian, which is the usual starting point of kinetic description, can be obtained by integrating out the bulk degrees freedom. In such a way the LG coupling constants appear to be explicitly dependent on the bulk properties of the adsorbed species (local density, pair correlation, etc).

Applying the association theory [16] allows us to treat an association-dissociation balance near the surfaces. Based on this we determine an effect of dissociation on adsorption and on surface diffusion.

Moreover, in this article we argue that all elementary processes (adsorption and dissociation, diffusion and dissociation) are mutually coupled through their connection with the bulk phase.

The paper is organized as follows. In section 2 we present a brief review of theoretical results concerning ER and LH kinetics. Coupling of interfacial properties with the fluid bulk is discussed in section 3 in terms of the sticky site model. Then in section 4 we apply the association theory to a description of adsorption and diffusion under the action of dissociation. Our conclusions are collected in section 5 .

\section{Mesoscopic kinetics within the ER and LH schemes}

Kinetics of catalytic reactions is usually described starting from the lattice gas (LG) description of the catalyst-gas system

$$
H=-\frac{1}{2} \sum_{i j, \alpha, \beta} J_{i j}^{\alpha \beta} n_{i}^{\alpha} n_{j}^{\beta}-\sum_{i, \alpha} V^{\alpha} n_{i}^{\alpha},
$$

where $i, j$ run over the lattice sites and $\alpha, \beta$ mark the species. The set of occupation numbers $n_{i}^{\alpha}=0,1$ controls the coverage of the sites. The one-site potential $V^{\alpha}$ describes an adatom interaction with the catalyst lattice and contains information about the ambient gas state through its dependence on the chemical potential. The coupling constant $J_{i j}^{\alpha \beta}$ describes the interaction between chemisorbed particles. The transition rates

$$
W_{i j}^{\alpha}\left(\left\{n_{l}\right\}\right)=\nu_{0}^{\alpha} \exp \left(-\beta V^{\alpha}-\beta \sum_{k, \beta} J_{i k}^{\alpha \beta} n_{k}^{\beta}\right) n_{i}^{\alpha}\left(1-n_{j}^{\alpha}\right)
$$

correspond to the jumps from site $i$ to site $j$ for a given species $\alpha$, with $\nu_{0}^{\alpha}$ being a frequency factor. By analogy one can define the rate $W^{\gamma, \alpha}\left(\left\{n_{l}\right\},\left\{n_{i}\right\}\right)$ for transition from a state with the set $\left\{n_{i}\right\}$ to a state with $\left\{n_{l}\right\}$.

Then the master equation

$$
\tau \frac{\mathrm{d} P^{\alpha}\left(\left\{n_{i}\right\}, t\right)}{\mathrm{d} t}=\sum_{\gamma} \sum_{\left\{n_{l}\right\}}\left[P^{\gamma}\left(\left\{n_{l}\right\}, t\right) W^{\gamma, \alpha}\left(\left\{n_{l}\right\},\left\{n_{i}\right\}\right)-P^{\alpha}\left(\left\{n_{i}\right\}, t\right) W^{\alpha, \gamma}\left(\left\{n_{i}\right\},\left\{n_{l}\right\}\right)\right]
$$


describes the evolution of the probability $P^{\alpha}\left(\left\{n_{i}\right\}, t\right)$ to find the system in a state $\left\{n_{i}\right\}$ at time $t$. Here $\tau$ gives a characteristic time scale. Constructing the transition rates $W$ according to (1) or (2) one obtains from (5) approximate kinetic equations for the species involved in the reaction. The most common way of constructing is the mean field approach (MFA) which underestimates the coverage fluctuations neglecting the correlation between them. Quite often a coarse grained mesoscopic description is introduced by averaging the master equation over the scales comparable with the lattice spacing. This allows us to explore general tendencies which are similar for the crystalline and for the amorphous catalytic surfaces.

\subsection{ER mechanism}

The ER scheme implies the adsorption of one species with the other species reacting directly from the gas phase. Following [7] we consider a single-component adsorbate to illustrate the essence. Particles may adsorb on the surface, desorb from it, and diffuse across it. In addition, the reaction occurs between adsorbed and gas particles. The products immediately leave the surface. The time evolution of the coverage $x$ is described by a mesoscopic kinetic equation

$$
\frac{\partial x}{\partial t}=k_{\mathrm{a}} p(1-x)-k_{\mathrm{d}} x-k_{r} x+\nabla\left[\frac{D}{k T}(1-x) x \nabla U(\mathbf{r})\right]+D \nabla^{2} x,
$$

where $k_{\mathrm{a}}, k_{\mathrm{d}}, k_{r}$ and $D$ are the adsorption, the desorption, the reaction and the diffusion coefficients, respectively; $p$ is the gas pressure; $T$ is the temperature, $k$ is the Boltzmann constant. In addition, a viscous flow of the adsorbate due to the gradient of the surface potential $U(\mathbf{r})$ is included. The potential is caused by lateral pairwise attractive interaction potential $u(\mathbf{r})$

$$
U(\mathbf{r})=-\int \mathrm{d} \mathbf{r}^{\prime} u\left(\mathbf{r}-\mathbf{r}^{\prime}\right) x\left(\mathbf{r}^{\prime}\right)
$$

The pair interaction was taken in the Gaussian form

$$
u(r)=\frac{u_{0}}{\pi r_{0}^{2}} \exp \left(-\frac{r^{2}}{r_{0}^{2}}\right),
$$

where $u_{0}$ and $r_{0}$ are the interaction strength and radius, respectively. Desorption coefficient is potential dependent

$$
k_{\mathrm{d}}=k_{\mathrm{d}}^{0} \exp (U(\mathbf{r}) / k T)
$$

with $k_{\mathrm{d}}^{0}$ being the desorption coefficient in the absence of interaction.

In the absence of diffusion the stationary state is given by a solution of

$$
\alpha \frac{1-x}{x}-\gamma=\exp (-\varepsilon x)
$$

where $\alpha=k_{\mathrm{a}} p / k_{\mathrm{d}}^{0}, \gamma=k_{r} / k_{\mathrm{a}} p, \varepsilon=u_{0} / k T$. Without the reaction contribution $\gamma=0$, equation (9) corresponds to the standard MFA result for the adsorption isotherm. 
For the critical values of the parameters $\varepsilon=\varepsilon_{\mathrm{c}}=4, \alpha=\alpha_{\mathrm{c}}=1 / e^{2}, x=x_{\mathrm{c}}=1 / 2$ the system exhibits a first-order phase transition from a dilute (gaseous) to a condensed (liquid-like) phase. The reaction shifts the critical parameters $\varepsilon_{\mathrm{c}}=4(1+\gamma), \alpha_{\mathrm{c}}=$ $1 /\left[e^{2}(1+\gamma)\right], x_{\mathrm{c}}=1 /[2(1+\gamma)]$. In other words, we have a coexistence of two stable states: some fraction of the surface is highly covered (droplets), while the rest is almost empty (holes). This bistability is essential because it allows one to draw the conclusion on how the reaction occurs. Since the production rate is proportional to $(1-x)$, we may conclude that due to the coupling of bistability and ER-like reaction, the system may exhibit a patterning. Namely, a highly reactive state around the holes and almost a poisoned state around the droplets. This is indeed the case, as it follows from numerical simulations [7]. Including the diffusion, these authors observed various modulated structures.

Of course, the one-component scheme illustrated above is too simple to be directly applied to the catalysis. For this purpose a generalization to multicomponent systems as well as a more accurate account for the kinetic coefficients are necessary.

\subsection{LH mechanism}

The LH scheme requires a more extensive description because we have to treat one more surface process. Since the product molecules immediately desorb, the acceleration rate correlates with the fractions of vacant sites $x_{\mathrm{v}}$ and with vacant nearest neighbour pairs $x_{v v}$. Then a simple stability analysis [8] provides a clear picture of the physics behind. The time evolution is given by

$$
\begin{aligned}
\frac{\mathrm{d} x_{\mathrm{v}}}{\mathrm{d} t} & =D_{\mathrm{v}} \nabla^{2} x_{\mathrm{v}}+r_{11} x_{\mathrm{v}}+r_{12} x_{v v}+O\left(x_{\mathrm{v}}^{2}, x_{v v}^{2}\right), \\
\frac{\mathrm{d} x_{v v}}{\mathrm{~d} t} & =D_{v v} \nabla^{2} x_{v v}+r_{21} x_{v}+r_{22} x_{v v}+O\left(x_{\mathrm{v}}^{2}, x_{v v}^{2}\right),
\end{aligned}
$$

where $D_{\mathrm{v}}, D_{v v}$ are the generalized diffusion coefficients and $r_{i j}$ is the matrix of kinetic coefficients (adsorption, desorption, reaction, etc). All these quantities are functions of the impingement rate $y$. For qualitative purposes it is enough to linearize these equations, neglecting the diffusion and higher order terms in $x_{\mathrm{v}}$ and $x_{v v}$. Then, choosing the linear combinations of $x_{\mathrm{v}}$ and $x_{v v}$, i.e., $x_{+}$and $x_{-}$, which diagonalize the $r_{i j}$ matrix, we have

$$
\begin{aligned}
& \frac{\mathrm{d} x_{+}}{\mathrm{d} t}=\lambda_{+} x_{+}, \\
& \frac{\mathrm{d} x_{-}}{\mathrm{d} t}=\lambda_{-} x_{-} .
\end{aligned}
$$

The existence of adsorbing or poisoned states may be discussed in terms of the eigenvalues $\lambda_{+}$and $\lambda_{-}$. Specifically for CO oxidation [8], $r_{21}=-r_{11} \propto y, r_{22} \propto$ $-(1-y)$ and $r_{12} \propto 2 y$. Diagonalization of this $r_{i j}$ matrix yields

$$
\lambda_{ \pm}=-\frac{1}{2} \pm \frac{1}{2} \sqrt{1-4 y+12 y^{2}} .
$$


It is easy to check that $\lambda_{+}$changes the sign at the critical value $y=y_{1}=1 / 3$, while $\lambda_{-}$is negative for all $y$. This implies the existence in this model at $y=y_{1}$ of a second-order phase transition from an absorbing state, wherein the surface is passivated (catalytically inactive or "poisoned") by one of the chemical constituents (O) to an active state. It was shown that this transition belongs to the universality class of Reggeon field theory or a direct percolation. As we will see below, this critical value $y_{1}=1 / 3$ is very close to the computer simulation result.

A more detailed description giving nontrivial results is the site approximation proposed by Dickman [9] in applying to $\mathrm{CO}$ oxidation. The catalyst was modelled as a square lattice; each site may be vacant or occupied by oxygen $(\mathrm{O})$ atom or a carbon monoxide molecule $(\mathrm{CO})$. $\mathrm{CO}$ arrive at the surface at a rate $y_{\mathrm{CO}}$ and, upon encountering a vacant site, immediately adsorb. $\mathrm{O}_{2}$ molecules arrive at the rate $y_{\mathrm{O}}=1-y_{\mathrm{CO}}$ and adsorb at a nearest-neighbour pair of vacant sites. Surface diffusion and desorption were ignored. The only dynamic process was the oxidation of $\mathrm{CO}$ to form $\mathrm{CO}_{2}$ which left the surface immediately. Based on this scheme the following equations of motion for surface concentrations $x_{\mathrm{a}}$ were obtained [9]

$$
\begin{aligned}
\frac{\mathrm{d} x_{\mathrm{O}}}{\mathrm{d} t} & =2 y_{\mathrm{O}} x_{\mathrm{v}}^{2}\left(1-x_{\mathrm{CO}}\right)^{3}-y_{\mathrm{CO}}\left[1-\left(1-x_{\mathrm{O}}\right)^{4}\right], \\
\frac{\mathrm{d} x_{C}}{\mathrm{~d} t} & =-2 y_{\mathrm{O}} x_{\mathrm{v}}^{2}\left[1-\left(1-x_{\mathrm{CO}}\right)^{3}\right]+y_{\mathrm{CO}} x_{\mathrm{v}}\left(1-x_{\mathrm{O}}\right)^{4}, \\
\frac{\mathrm{d}\left(x_{\mathrm{O}}-x_{\mathrm{CO}}\right)}{\mathrm{d} t} & =2 y_{\mathrm{O}} x_{\mathrm{v}}^{2}-y_{\mathrm{CO}} x_{\mathrm{v}},
\end{aligned}
$$

where $x_{\mathrm{v}}$ is the fraction of vacant sites. The system of this equations yields at $y=y_{2}$ a first-order phase transition from the reactive for $y<y_{2}\left(x_{v} \neq 0\right)$ to CO poisoned state for $y>y_{2}\left(x_{v}=0\right)$. As we will see below, the value $y_{2}=0.4787$ is very close to the computer simulation result. But the second-order transition from $\mathrm{O}$ poisoned to the reactive state obtained from the stability analysis was not found in this approach.

Since the shortcoming of the site approximation appears to be largely due to the neglect of the prohibition against O-CO nearest-neighbour pairs, Dickman [9] derived the kinetic equations for the concentrations in pair approximation, which is next in a series of progressively more accurate cluster methods. This allowed us to refine the theory and improve an agreement with computer simulation results [4] for the ZGB scheme. The steady state phase diagram is given in figure 1 . For $0<y_{\mathrm{CO}}<$ $y_{1}=0.389$ the catalyst is "poisoned" with oxygen, while for $y_{\mathrm{CO}}>y_{2}=0.525$ it is poisoned with $\mathrm{CO}$. The poisoning means that the catalyst is mainly covered by one of the species. The reactive state exists in a window $y_{1}<y_{\mathrm{CO}}<y_{2}$ where a nonzero rate of $\mathrm{CO}_{2}$ production is observed. Note that $\mathrm{O}$ poisoning is a second-order (continuous) transition, while CO poisoning is a first-order (discontinuous) kinetic transition. In order to gain insight into this reaction Luque studied [10] the distribution of adsorbed pairs and their effect on the standard ZGB scheme. This theory is a refinement of the pair approximation by Dickman [9] to counting the orientation of pairs at a square lattice. The system was analyzed in terms of monomeric $\left(n_{\mathrm{O}}, n_{\mathrm{CO}}\right)$ and pair $\left(n_{\mathrm{CO}-\mathrm{CO}}, n_{\mathrm{CO}-\mathrm{O}}, n_{\mathrm{O}-\mathrm{O}}\right)$ concentrations. The transitions probabilities $P_{i}$ correspond 
to: $i=1$, adsorption of $\mathrm{CO} ; i=2$, adsorption of $\mathrm{O}_{2} ; i=3$, desorption of $\mathrm{CO}_{2}$. The evolution equations are

$$
\begin{aligned}
\frac{\mathrm{d} n_{\mathrm{CO}}}{\mathrm{d} t}= & n_{5}[\mathrm{~V}]-4 n_{8}[\mathrm{CO}-\mathrm{O}] \\
\frac{\mathrm{d} n_{\mathrm{O}}}{\mathrm{d} t}= & 4 n_{8}[\mathrm{~V}-\mathrm{V}]-4 n_{8}[\mathrm{CO}-\mathrm{O}] \\
\frac{\mathrm{d} n_{\mathrm{CO}-\mathrm{CO}}}{\mathrm{d} t}= & 2 n_{5}[\mathrm{CO}-\mathrm{V}]-6 n_{8}[\mathrm{CO}-\mathrm{CO}-\mathrm{O}] \\
\frac{\mathrm{d} n_{\mathrm{CO}-\mathrm{O}}}{\mathrm{d} t}= & n_{5}[\mathrm{O}-\mathrm{V}]-3 n_{8}[\mathrm{CO}-\mathrm{V}-\mathrm{V}]-n_{8}[\mathrm{CO}-\mathrm{O}] \\
& -3 n_{8}[\mathrm{O}-\mathrm{CO}-\mathrm{O}]-3 n_{8}[\mathrm{CO}-\mathrm{O}-\mathrm{CO}] \\
\frac{\mathrm{d} n_{\mathrm{O}-\mathrm{O}}}{\mathrm{d} t}= & n_{8}[\mathrm{~V}-\mathrm{V}]+6 n_{8}[\mathrm{O}-\mathrm{V}-\mathrm{V}]-6 n_{8}[\mathrm{O}-\mathrm{O}-\mathrm{CO}]
\end{aligned}
$$

where

$$
\begin{aligned}
n_{5}[\mathrm{~V}] & =n\left[\begin{array}{ccc}
\times & \times & \\
\times & \mathrm{V} & \times \\
& \times &
\end{array}\right] P_{1}, \\
n_{5}[z-\mathrm{V}] & =n\left[\begin{array}{ccc}
\times & \\
z & \mathrm{~V} & \times \\
& \times &
\end{array}\right] P_{1}, \\
n_{8}[r-s] & =n\left[\begin{array}{cccc}
\times & \times & \times & \\
\times & \times & \times
\end{array}\right] P_{i}, \\
n_{8}[z-r-s] & =n\left[\begin{array}{cccc} 
& \times & \times &
\end{array}\right] P_{i},
\end{aligned}
$$

with the notation

$$
n\left[\begin{array}{ccc} 
& \times & \\
z & \mathrm{~V} & \times \\
& \times &
\end{array}\right]
$$

used for the density of five-site clusters which have got a vacant site in the center;

$$
n\left[\begin{array}{cccc} 
& \times & \times & \\
\times & r & s & \times \\
& \times & \times &
\end{array}\right]
$$

denotes the density of eight-site clusters whose center is occupied by CO-O, O$\mathrm{CO}$, or $\mathrm{V}-\mathrm{V}$. The symbol $\times$ represents indistinctly $\mathrm{CO}, \mathrm{O}$, and $\mathrm{V}$ (vacancy). $\mathrm{A} \times$ substituted by $z$ means that the site can be occupied by a monomer (CO or O). $P_{i}$ in (25) and (26) is $P_{2}$ for $r-s=v-v$ and $P_{3}$ for $r-s=\mathrm{CO}-\mathrm{O}$ or O-CO. To solve the evolution equations the following closure approximations were used

$$
n\left[\begin{array}{lll} 
& 1 & \\
2 & V & 4 \\
& 3 &
\end{array}\right]=\left(n_{v-1} n_{v-2} n_{v-3} n_{v-4}\right) / n_{\mathrm{v}}^{3},
$$




$$
n\left[\begin{array}{rrrr}
1 & 6 & \\
2 & r & s & 5 \\
& 3 & 4 &
\end{array}\right]=\left(n_{r-1} n_{r-2} n_{r-3} n_{r-s} n_{s-4} n_{s-5} n_{s-6}\right) /\left(n_{r}^{3} n_{\mathrm{s}}^{3}\right)
$$

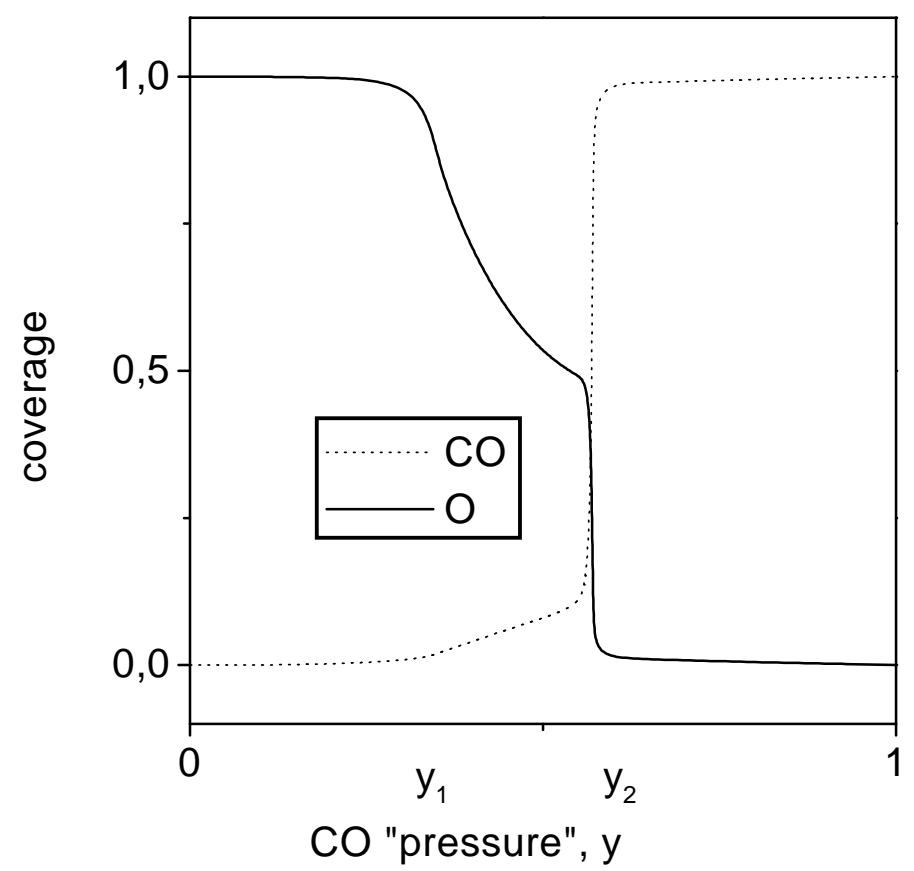

Figure 1. Steady state phase diagram for the ZGB scheme of catalytic CO oxidation (schematically). The impingement rates $y_{1}$ and $y_{2}$ indicate the boundaries of the second- and first order kinetic transitions between the reactive and poisoned states. The reactive state is in between $y_{1}$ and $y_{2}$.

Four zones were found for the steady state

(1) $0<y_{\mathrm{CO}}<0.3310$ : the catalyst is poisoned by $\mathrm{O}_{2}$.

(2) $0.3310<y_{\mathrm{CO}}<0.3849$ : a reactive steady state.

(3) $0.3849<y_{\mathrm{CO}}<0.5692$ : three steady states, one is unstable and the others are stable. One stable solution is a reactive steady state, but in the other one the catalyst is poisoned by $\mathrm{CO}$.

(4) $0.5692<y_{\mathrm{CO}}<1$ : the catalyst is poisoned by CO.

The scheme outlined above serves as a basis for a description of the catalytic oxidation of CO. Further extensions were directed towards the inclusion of spatial correlation between adsorbed particles (diffusion, pair interactions, etc). The role of the surface diffusion within the ZGB scheme was analyzed [11] employing the computer simulation and the mean field theory. It was found that $y_{1}$ shifts to lower values and $y_{2}$ shifts to higher values with the increasing diffusion coefficient. It is concluded that the nature of poisoning transitions is not altered by diffusion. The effects of the interaction between adatoms was considered in [12], choosing the transition probabilities in the Arrhenius form.

$$
P_{i}=A_{i} \exp \left(-\frac{E_{a}^{i}}{k T}\right)
$$


where $A_{i}$ are frequency factors, $E_{\mathrm{a}}^{i}$ are activation energies of each of the three elemental processes. The activation energy is divided into two additive terms, corresponding to the action of the substrate and to the interaction between the nearest adatoms, respectively

$$
E_{\mathrm{a}}^{i}=E_{i}+\sum_{j k} n_{j k} E_{j k}^{v}(i=1,2), \quad E_{a}^{3}=E_{3}+\sum_{j k} n_{j k} E_{j k}^{\prime}
$$

where $j, k=\mathrm{CO}$, O represents all the bonds that may be formed between monomers that participate in the process and their possible neighbour monomers. For $i=1$ $j=\mathrm{CO}$, for $i=2 j=\mathrm{O}$ and for $i=3 j=\mathrm{CO}, \mathrm{O} . E_{i}$ is the contribution of the substrate to the total activation energy connected with the second term in the hamiltonian (3); $E_{j k}$ and $E_{j k}^{\prime}$ are the contribution to the total activation energy owing to the interaction between the nearest-neighbour adatoms connected with the first term in the hamiltonian (3), $n_{j k}$ is the pair number of the type $(j, k)$. For the sake of simplicity, it was assumed that

$$
E_{j k}^{\prime}=-E_{j k}
$$

Consequently, it was shown that the rate of $\mathrm{CO}_{2}$ production increases when repulsive $\mathrm{CO}-\mathrm{CO}$ and $\mathrm{O}-\mathrm{O}$ interactions together with the attractive $\mathrm{CO}-\mathrm{O}$ interaction are taken into account. The interval associated with the $\mathrm{CO}_{2}$ production becomes wider than in the case without interaction.

Recently [13] the system of equations (18)-(22) was supplemented by the equation for the rate of change of the surface temperature

$$
C \frac{\mathrm{d} T}{\mathrm{~d} t}=-L\left(T-T_{\mathrm{B}}\right)+\sum_{i=1}^{3} \Delta H_{i} P_{i}
$$

where $C$ is the heat capacity of the system, $L / C$ is the relaxation rate of $T$ to $T_{B}$ being room temperature, $\Delta H_{i}$ are the reaction heats of processes (1).

Thus, the bistability and oscillations of temperature and concentrations were observed. It was shown that an attractive interaction between $\mathrm{CO}-\mathrm{CO}$ and $\mathrm{O}-\mathrm{O}$ pairs and repulsive interactions between $\mathrm{CO}-\mathrm{O}$ pairs produce similar behaviours, increasing the oscillation amplitudes of temperature and concentration of CO-O pairs. Thus, the oscillations of CO-O pairs are facilitated and $\mathrm{CO}_{2}$ production is favoured. In this way, oscillations are favoured with respect to the case where interaction is not considered.

\section{Coupling of interfacial properties with the fluid bulk}

In order to gain a quantitative information the scheme above should be improved in (at least) two ways. First of all, it is necessary to go beyond the mean-field theory towards developing more refined kinetic equations. Secondly, a more accurate description of the rates of elementary processes is required. Especially important is 
to make a connection between the rates and particle properties in a bulk phase. This can be done in the framework introduced in $[14,15]$.

For simplicity we consider a one-component fluid with a pair interaction $U_{2}=$ $\sum_{i j} U\left(\mathbf{r}_{i}, \mathbf{r}_{j}\right)$ and bulk number density $\rho$. As we shall see hereinafter, the pair potential may include various interactions (hard core repulsion, short range attraction, etc). The fluid is near a hard wall (located at $z=0$ and modelled by the potential $\left.U_{\mathrm{hw}}(z)\right)$ on which there is a lattice of sticky sites (lattice spacing is $d$ ), modelling a crystalline structure of a catalyst. The adsorption potential $U_{\mathrm{s}}\left(\mathbf{r}_{i}\right)$ is defined by

$$
\exp \left(-\beta U_{\mathrm{s}}\left(\mathbf{r}_{i}\right)\right)=1+\lambda \sum_{\mathbf{R}_{\mathrm{m}}} \delta\left(\mathbf{R}_{i}-\mathbf{R}_{\mathrm{m}}\right) \delta(z)
$$

where $\mathbf{R}_{i}$ is a projection of $\mathbf{r}_{i}$ onto the wall, $\mathbf{R}_{\mathrm{m}}$ is a position of a lattice site and $\lambda$ is the stickiness parameter. This singular one-body potential allows one to perform an exact integration in the partition function

$$
Z=Z_{\text {ref }} \sum_{n=0} \frac{\lambda^{n}}{n !} \sum_{\mathbf{R}_{n}} \rho_{n}^{\mathrm{ref}}\left(\mathbf{R}_{1}, \ldots, \mathbf{R}_{n}\right),
$$

where $Z_{\text {ref }}$ is the partition function for the same system but without the adsorbing potential, and $\rho_{n}^{\mathrm{ref}}\left(\mathbf{R}_{1}, \ldots, \mathbf{R}_{n}\right)$ is the $n$-body distribution function taken at the positions of the lattice sites. In such a way we have an infinite series on $\lambda$ including the correlations of all orders for the reference state. If only pair interactions are important then the problem can be mapped onto the lattice gas model $[14,15]$

$$
\Xi=Z / Z_{\text {ref }} \sum_{t_{i}} \exp \left(-\beta H_{\mathrm{LG}}\right)
$$

with the Hamiltonian

$$
H_{\mathrm{LG}}=\sum_{i j} W\left(\mathbf{R}_{i}, \mathbf{R}_{j}\right) t_{i} t_{j}-\sum_{i} \mu\left(\mathbf{R}_{i}\right) t_{i}
$$

which is the same as (3) but now the chemical potential $\mu\left(\mathbf{R}_{i}\right)$ and pair interaction $W\left(\mathbf{R}_{i}, \mathbf{R}_{j}\right)$ are closely connected with the properties of the fluid in the bulk phase. Namely,

$$
\begin{aligned}
\beta \mu\left(\mathbf{R}_{i}\right) & =\ln \left(\lambda \rho_{1}^{\mathrm{ref}}\left(\mathbf{R}_{i}\right)\right), \\
\beta W\left(\mathbf{R}_{i}, \mathbf{R}_{j}\right) & =-\ln \left(g_{2}^{\mathrm{ref}}\left(\mathbf{R}_{i}, \mathbf{R}_{j}\right)\right),
\end{aligned}
$$

where $\rho_{1}^{\text {ref }}\left(\mathbf{R}_{i}\right)$ and $g_{2}^{\text {ref }}\left(\mathbf{R}_{i}, \mathbf{R}_{j}\right)$ are respectively the one-body and pair correlation functions for the reference state (i.e., a state without specific adsorption). This representation implies that not only adsorption [14] but all other transition rates (4) for two-dimensional processes should depend on the properties of the third dimension.

As we have mentioned above, the reference correlation functions describe specific features of the adsorbed particles, such as equation of state or associationdissociation balance near the surface. The latter point is of practical importance since the adsorption is often dissociative $\left(\mathrm{O}_{2}\right.$ or $\left.\mathrm{N}_{2}\right)$. Choosing properly the reference state we may use an information on $\rho_{1}^{\text {ref }}\left(\mathbf{R}_{i}\right)$ and $g_{2}^{\text {ref }}\left(\mathbf{R}_{i}, \mathbf{R}_{j}\right)$ as an input for 
calculating the surface properties. Since a flat wall problem is translationally invariant along the surface, we are interested in the contact values $\rho_{1}^{\text {ref }}(0)$ and $g_{2}^{\text {ref }}(\sigma)$, provided that the lattice spacing $d$ is of the order of $\sigma$. Generalization of this scheme to multicomponent systems is straightforward.

\section{Application of the associative theory to the description of adsorption and diffusion}

Association theory [16] is a powerful tool for describing various complexation processes and their effect on adsorption. This approach is successfully used for predicting equilibrium properties of monomer-dimer mixtures [17], polymer chains [18] or network-forming fluids [19]. For spatially inhomogeneous systems [20] the theory gives quantitatively correct predictions [21] if compared to computer simulations and a real experiment [22].

\subsection{Dimerizing hard spheres as a model of association and dissociation}

It is known that a dissociative adsorption of dimers is one of the intermediate steps in catalytic reactions. Therefore, a description of association-dissociation equilibrium near adsorbing surfaces is desirable. For this purpose we use a dimerizing hard sphere (DHS) model [17]. The reference pair potential is a sum of hard-sphere repulsion and off-center associative attraction. This saturable interaction is responsible for the formation of dimers. Therefore, we treat an association-dissociation equilibrium of a hard sphere fluid. In extreme cases of vanishing or very strong association we deal with a monomers or hard-sphere dumbbells. Bulk properties of this system were investigated within the thermodynamic perturbation theory [17] and integral equation approach [23]. A density variation near a hard wall was discussed in our previous study [20].

The wall-particle and pair contact probabilities are

$$
\begin{aligned}
\rho_{1}^{\mathrm{ref}}(0) & =\rho\left[\frac{1+2 \eta}{(1-\eta)^{2}}-\frac{x_{\mathrm{d}}}{2(1-\eta)}\right] \\
g_{2}^{\mathrm{ref}}(\sigma) & =\frac{1+\eta / 2}{(1-\eta)^{2}}-\frac{x_{\mathrm{d}}}{2(1-\eta)}+C \frac{x_{\mathrm{d}}^{2}}{\eta},
\end{aligned}
$$

where $\eta=\pi \rho \sigma^{3} / 6$ is the bulk packing fraction and $x_{\mathrm{d}}$ is the fraction of dimers with $x_{0}=1-x_{\mathrm{d}}$ being the fraction of monomers (dissociated molecules). The latter is determined by a mass action law

$$
x_{0}+x_{0}^{2} \rho g(\sigma) K=1 .
$$

Here $K$ gives a strength of association (it could be related to the dissociation energy of a dimer) and $g(\sigma)$ is the monomer-monomer contact value (the first term in (40)). Notice, that in equation (40) we have introduced an "intramolecular" term $C x_{\mathrm{d}}^{2} / \eta$ which corrects the contact probability obtained within the Percus-Yevick 
(PY) approximation. The latter underestimates the intramolecular correlation. Such a correction is necessary to account for a fact that the probability of two molecules to be in contact should be high even at a low density, if $x_{\mathrm{d}}$ is nonzero. Since the correction is done in ad hoc manner, we cannot set the value of a constant $C$. It can be estimated from a requirement of coinciding contact values coming from (40) in the limit of complete dimerization $x_{\mathrm{d}} \rightarrow 1$ and the one coming from a hard- sphere chain model [18] in the limit when the mean chain length equals 2 . This gives $C=1 / 96$. Nevertheless, this value of $C$ results from the PY+ideal chain approximation [18], and consequently is underestimated. For that reason we set $C=1$ without loss of generality, since this choice does not affect our qualitative conclusions.

\subsection{Adsorption and dissociation}

It is known [24] that a mechanism of the dimer adsorption is an important factor in surface reactions. Discussing an interplay between the adsorption and dissociation we may distinguish between two cases. A dimer may be adsorbed without dissociation or it has to dissociate prior to adsorption. In both cases the lateral interaction between adsorbates as well as their chemical potential should manifest an effect of a dissociation-association balance near the surface. Consequently, this effect should be seen in other kinetic coefficients through their dependence on the chemical potential and lateral interactions.

\subsubsection{Associative adsorption}

The first situation was analyzed in our recent paper [25]. Nevertheless, there we were mainly focused on the intermolecular correlations (the contact value (40) was without the third term). Taking into account the intra- part, we may draw the same conclusion as for short chains [21]. Namely, the LG Hamiltonian (36) exhibits criticality when the coupling constants $-\beta W=\ln \left(g_{2}^{\text {ref }}(\sigma)\right)$ and $\beta \mu=\ln \left(\lambda \rho_{1}^{\text {ref }}(0)\right)$ reach some critical values. For attractive interactions $\left(g_{2}^{\text {ref }}(\sigma)>1\right)$ the critical behaviour is associated with the liquid-gas coexistence at the surface. Rigorous estimation [15] for hexagonal lattices gives

$$
\left.g_{2}^{\mathrm{ref}}(\sigma)\right|_{\mathrm{c}}=3,\left.\quad \lambda \rho_{1}^{\mathrm{ref}}(0)\right|_{\mathrm{c}}=1 / 27 .
$$

Since $g_{2}^{\text {ref }}(\sigma)$ given by equation (40) is a nonmonotonic function of density, first of these conditions can be satisfied at two different values of $\eta$.

One is rather high: $\eta_{1}=0.3713$ for hard spheres and $\eta_{1}=0.442$ for dimers). It corresponds to an effective entropic attraction induced by packing effects. This point is almost insensitive to the level of association. The existence of this kind of critical behaviour suggests that all other kinetic coefficients should exhibit an abrupt change when the adsorbate becomes dense enough.

The other critical density $\eta_{2}$ is low: $\eta_{2}=0.004$ for hard-sphere chains with the mean chain length equal 2). This point is governed mainly by the intra-molecular correlation (the last term in equation (40)). At this domain the criticality is induced by the change of dimer orientation. At very low densities $\left(\eta<\eta_{2}\right)$ the dimers 
are adsorbed in parallel to the surface, while a perpendicular orientation becomes preferential at $\eta>\eta_{2}$. The low-density critical point is extremely sensitive to the dimerization degree (it vanishes if $x_{\mathrm{d}} \rightarrow 0$ ). This implies that even at very low densities near the surface a correlation through the fluid bulk is not negligible. Adsorption (and all other kinetic coefficients) become strongly dependent upon the rate of dissociation. This is contrary to the standard kinetic approaches which ignore such a dependence.

\subsubsection{Dissociative adsorption}

Now we turn the attention to the second case (dissociative adsorption). Interestingly, that we can get an exact solution, provided that the properties of a completely dissociated systems (monomers near an adsorbing lattice $[14,15]$ ) are known. The partition function of the problem under consideration can be written in the following form

$$
Z=\frac{1}{N !} \int(\mathrm{d} \mathbf{r}) \mathrm{e}^{-\beta U_{0}} \prod_{i}\left[1+f_{\mathrm{st}}(i)\right] \prod_{i, j}\left[1+f_{\mathrm{as}}(i, j)\right]
$$

where $U_{0}$ denotes the potential of hard spheres near a hard wall, and $(d \mathbf{r})=\prod d \mathbf{r}_{i}$. The values of

$$
f_{\mathrm{st}}(i)=\exp \left\{-\beta U_{\mathrm{s}}(i)\right\}-1
$$

and

$$
f_{\text {as }}(i, j)=\exp \left\{-\beta U_{\text {as }}(i, j)\right\}-1
$$

are the Mayer functions for the lattice and association potentials, respectively.

Expanding the second product according to

$$
\prod_{i, j}\left[1+f_{\mathrm{as}}(i, j)\right]=1+\sum_{i, j} \prod_{l, m=1}^{i, j} f_{\mathrm{as}}(l, m)
$$

we extract the partition function $Z_{0}^{l}$ of the hard spheres near the lattice:

$$
Z_{0}^{l}=\frac{1}{N !} \int(\mathrm{d} \mathbf{r}) \mathrm{e}^{-\beta U_{0}} \prod_{i}\left[1+f_{\mathrm{st}}(i)\right]
$$

This contribution is identical to that derived in $[14,15]$, since the intermolecular site-site interaction is excluded. Then the partition function can be written as

$$
Z=Z_{0}^{l}+\frac{1}{N !} \int(\mathrm{d} \mathbf{r}) \mathrm{e}^{-\beta U_{0}} \prod_{i}\left[1+f_{\mathrm{st}}(i)\right] \sum_{i, j} \prod_{l, m=1}^{i, j} f_{\mathrm{as}}(l, m) .
$$

Expanding the product with $f_{\text {st }}(i)$ after some rearrangement we have

$$
Z=Z_{0}^{l}+Z_{\mathrm{as}}^{0}+Z_{\mathrm{as}}^{l}-Z_{0}^{0},
$$

where

$$
Z_{\mathrm{as}}^{0}=\frac{1}{N !} \int(\mathrm{d} \mathbf{r}) \mathrm{e}^{-\beta U_{0}} \prod_{i, j=1}\left[1+f_{\mathrm{as}}(i, j)\right]
$$


is the partition function of the associative subsystem near a hard wall, and

$$
Z_{\mathrm{as}}^{l}=\frac{1}{N !} \int(\mathrm{d} \mathbf{r}) \mathrm{e}^{-\beta U_{0}} \sum_{i} \prod_{l} f_{\mathrm{st}}(l) \sum_{i, j} \prod_{l, m=1}^{i, j} f_{\mathrm{as}}(l, m) \sum_{j} \prod_{\mathrm{m}} f_{\mathrm{st}}(m)
$$

is the contribution describing the coupling between the association and adsorption. The value $Z_{0}^{0}$ stands for the partition function of hard spheres near a hard wall.

We consider the case without the coupling contribution, i.e. $Z_{\mathrm{as}}^{l}=0$ due to the requirement that only one bond at any site (at the surface or molecule) is allowed. Thus, a given particle can be either dimerized or adsorbed. Then the free energy excess is given by

$$
F=F_{0}^{l}-F_{0}^{0}-\frac{1}{\beta} \ln \left[1+\left(\mathrm{e}^{-\beta\left(F_{\mathrm{as}}^{0}-F_{0}^{0}\right)}-1\right) \mathrm{e}^{\beta\left(F_{0}^{l}-F_{0}^{0}\right)}\right],
$$

where the free energies of unperturbed subsystems are as follows

$$
\begin{aligned}
\beta\left(F_{\mathrm{as}}^{0}-F_{0}^{0}\right) & =\ln x_{0}-\frac{x_{0}}{2}+\frac{1}{2}, \\
\beta\left(F_{0}^{l}-F_{0}^{0}\right) & =\frac{q \Theta_{0}^{2}}{2} W+\ln \left(1-\Theta_{0}\right) .
\end{aligned}
$$

The latter equation is written in the mean field approximation, with $\Theta_{0}$ and $q$ being the surface coverage (for fully dissociated particles) and coordination number of the lattice, respectively. Here $W=\ln [g(\sigma)]$ is the pair interaction between adsorbed monomers. In the "sticky" limit for association $f_{\text {as }}(i, j)=K \delta(r-\sigma)$ the fraction of monomers, $x_{0}$ is given by a mass action law (41), which predicts the rate of dissociation in the absence of adsorption.

The fraction of the wall sites occupied or the adsorption isotherm $\Theta$ can be calculated as

$$
\Theta=-\beta \lambda \frac{\partial\left(F-F_{0}^{0}\right)}{\partial \lambda}=\frac{\Theta_{0}}{1+\left(\mathrm{e}^{-\beta\left(F_{\mathrm{as}}^{0}-F_{0}^{0}\right)}-1\right) \mathrm{e}^{\beta\left(F_{0}^{l}-F_{0}^{0}\right)}},
$$

where $\Theta_{0}$ is the adsorption isotherm for hard-sphere monomers. This value is determined by $F_{0}^{l}-F_{0}^{0}$ and is calculated in [14]

$$
\frac{\Theta_{0}}{1-\Theta_{0}}=\lambda^{*} \exp \left(\beta q W \Theta_{0}\right)
$$

where $W$ and $\lambda^{*}=\exp (-\beta \mu)$ are density dependent parameters describing the lateral interaction between adsorbed particles and their effective chemical potential.

The coverage $\Theta$ as a function of the coverage $\Theta_{0}$ for fully dissociated monomers is displayed in figure 2. As expected, we have a linear dependence when $x_{\mathrm{d}}=0$. Note that $\Theta_{0}$ is considered as a measure of surface activity. This quantity, however, could be a nonmonotonic function of the stickiness $\lambda$ when the interaction $W$ becomes strong enough (either attractive or repulsive). At nonzero association $x_{\mathrm{d}} \neq 0$ the 


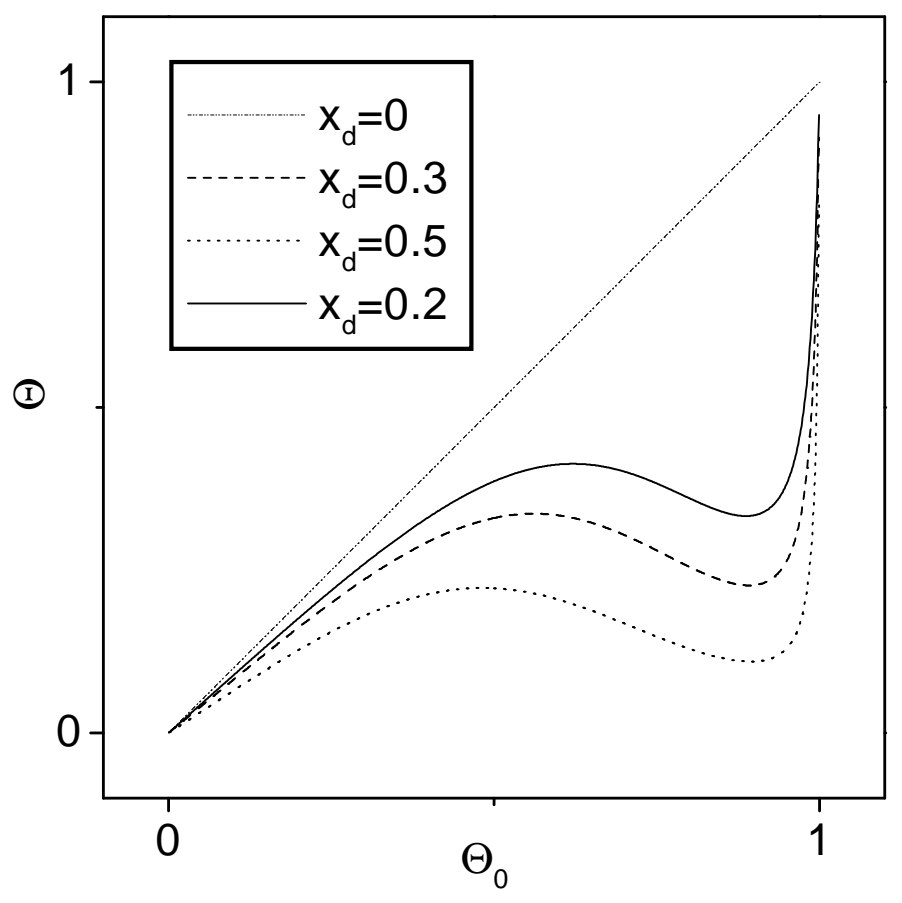

Figure 2. The dissociative adsorption coverage $\Theta$ as a function of the coverage $\Theta_{0}$ for a fully dissociated monomers. The curves correspond to a different dimerization degree indicated. The interaction is repulsive $\beta W=3$.

coverage grows much slower than $\Theta_{0}$ does. In case of repulsive interaction a nonmonotonic behaviour results from a competition between the surface activity and interparticle repulsion.

Of course, the dissociation rate is unchanged if (49), (41) refer to a seminfinite system. Nevertheless, if we assume that dissociation takes place in a narrow layer near the surface, then the reactive fluid is effectively two-dimensional. In this case the monomeric fraction $x_{0}$ and $\Theta$ are connected by the requirement of constant number density

$$
x_{0}+x_{0}^{2} \tilde{K}=1-\Theta,
$$

where $\tilde{K}=\rho g(\sigma)$ as before (see equations (41)). Note however that now $x_{0}$ is the fraction of nonadsorbed monomers and the association constant $K$ may differ from that in (41) because a local density near the surface is not the same as in the bulk fluid. For simplicity we neglect the latter difference and combine (51) and (53) to obtain a nonlinear equation for $x_{0}$. Solving this equation we have $x_{0}$ as a function of adsorption parameters. Based on this we discuss the change in the dissociation rate under the effect of adsorption. The fraction of nonadsorbed monomers is displayed in figure 3 as a function of surface activity. At extremely weak adsorbing potential $(\lambda \approx 0) x_{0}$ starts from a value determined by the bulk association constant $K$. The fraction $x_{0}$ falls down with the increasing surface activity. Repulsive lateral interaction between the monomers contributes to a nonmonotonic decrease of $x_{0}$. 


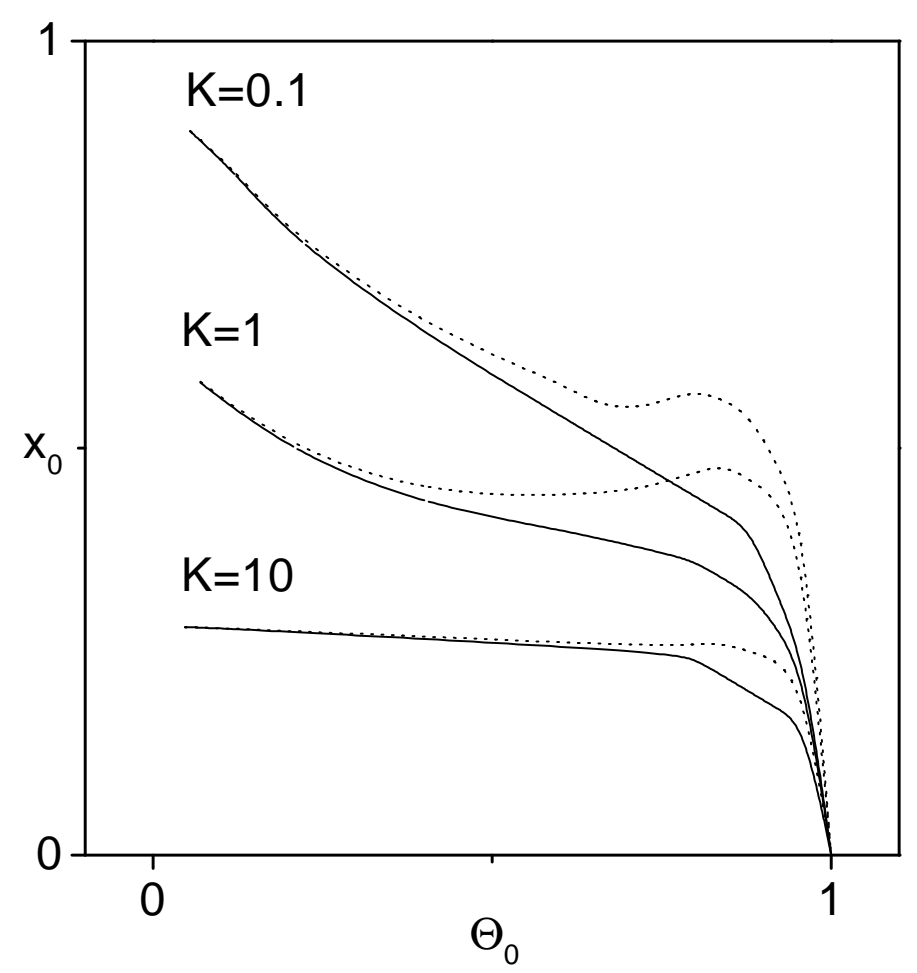

Figure 3. The fraction of nonadsorbed monomers as a function of coverage $\Theta_{0}$ at a different association constant $K$. Solid and dotted lines correspond to $\beta W=1$ and $\beta W=2$, respectively.

\subsection{Surface diffusion and dissociation}

Similar to the adsorption, the diffusion coefficient should also depend on the dissociation rate near the surface. Indeed, the diffusion coefficient $D$ for the LG model is defined as [26]

$$
D=d^{2} \Gamma(\Theta) \frac{q}{4} \frac{\partial \mu}{\partial \Theta}
$$

where $\Gamma(\Theta)$ is the rate of jumps to an adjacent site. Note that $\partial \Theta / \partial \mu$ is the compressibility which becomes infinite in the neighbourhood of the critical point. Therefore, $D$ exhibits deep minima when $\Theta$ as a function of $\mu$ makes steps (e.g. in the neighbourhood of a phase transition). Since $\Gamma(\Theta)$ itself has a nontrivial coverage dependence, an attempt was made [27] to provide a simple representation of the diffusion coefficient in terms of $\mu, W$ and $\Theta$. The following expression was obtained for attractive lateral interactions

$$
D=D_{0} \exp (-\beta \mu-q \Theta[\exp (\beta W)-1])
$$

with $D_{0}$ corresponding to the diffusion at low coverages. In our case $\mu$ and $W$ are the functions of the dissociation rate $x_{\mathrm{d}}$ (according to equations (37)-(40)). Specifically, we analyze the situation when adsorption of monomers and dimers is allowed (associative adsorption). For simplicity, the coverage $\Theta$ was assumed to be 
an independent parameter. This can be achieved by tuning $\lambda$ in accordance with variations of $\eta$ and $x_{\mathrm{d}}$.

In figure 4 the diffusion coefficient is plotted as a function of a dimeric fraction. As expected the diffusion decreases with the increasing density. $D$ interpolates between the diffusion coefficient for hard-sphere monomers $\left(\right.$ at $x_{\mathrm{d}}=0$ ) and that of dimers $\left(\right.$ at $x_{\mathrm{d}}=1$ ). But the behaviour is not monotonic. The maximum at low $x_{\mathrm{d}}$ is a consequence of the intra-molecular correlations at low densities. The maximum becomes more pronounced with the decreasing density. This is clear from figure 5 where $D / D_{0}$ is plotted as a function of $\eta$. The diffusion decreases with the increasing fraction of dimers. Simultaneously, the anomalous low diffusion is observed at low densities as $x_{\mathrm{d}}$ increases. This is again a consequence of the low-density critical behaviour. As discussed above the diffusion goes to zero when the system approaches the critical point (either at low or at high densities).

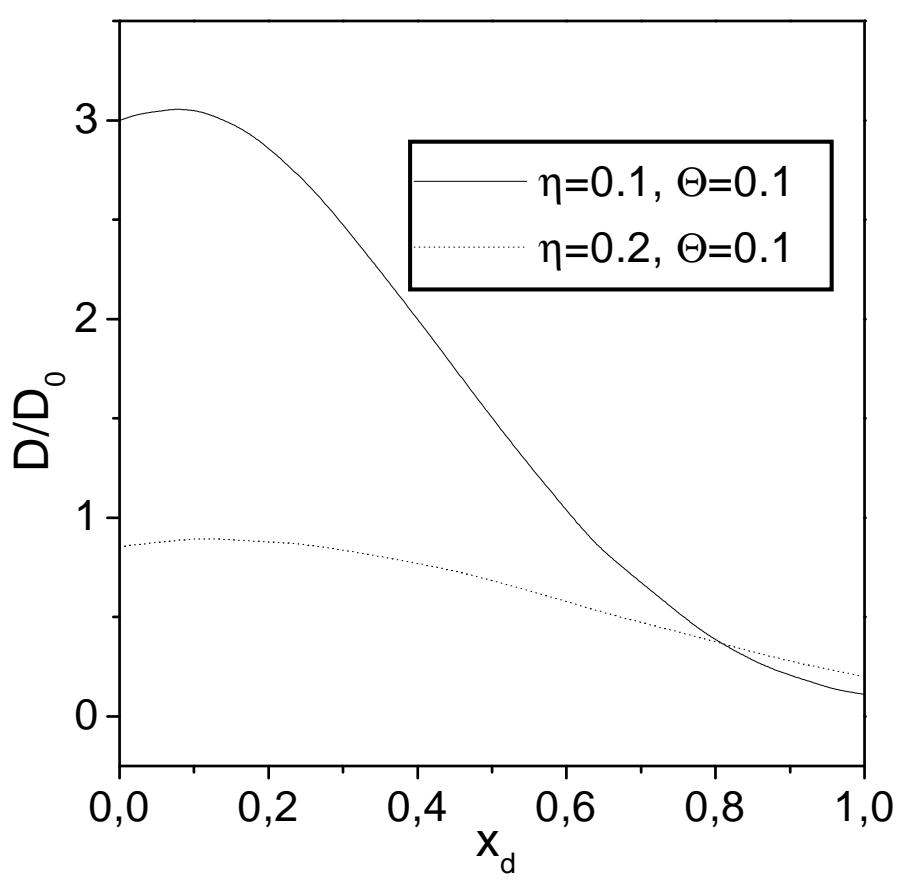

Figure 4. Diffusion coefficient as a function of the dimer fraction at different densities and coverages.

\section{Conclusion and overview}

A short review of theoretical results on the Langmuir-Hinschelwood and EleyRideal reaction schemes is presented.

In this paper we argue that the transition rates of surface elementary processes (adsorption, desorption, diffusion, etc) are coupled through the bulk properties of adsorbing species. This concerns the dependence of the chemical potential on the association-dissociation balance and an adsorbate density near the surface. 


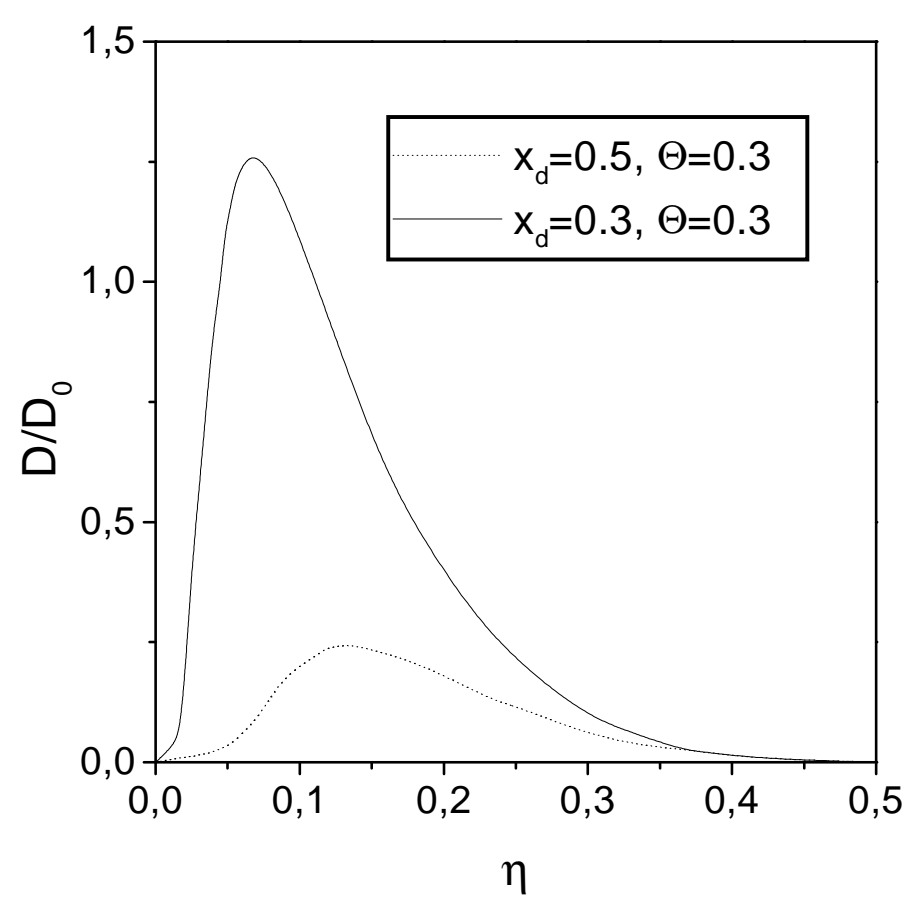

Figure 5. Diffusion coefficient as a function of density at different $x_{\mathrm{d}}$ and $\Theta$.

Adsorption is shown to be sensitive to a mechanism of dimer adsorption (associative or dissociative). Dissociative adsorption gives a nonmonotonic behaviour of the coverage with the surface activity. Simultaneously, the dissociation degree increases with the increasing adsorption. The associative adsorption implies the existence of a low-density critical point around which the adsorption isotherms are discontinuous. This causes an abnormal low surface diffusion at low densities. Implementation of these results to kinetic properties is briefly discussed.

As we have seen, the transition from a poisoned to a reactive state proceeds through a kinetic phase transition (first- or second-order). A connection between the equilibrium criticality (coverage as a function of chemical potential) and kinetic phase transitions remains to be investigated.

\section{References}

1. Somorjai G.A. // Chem. Rev., 1996, vol. 96, p. 1223.

2. Goodman D.W. // J. Phys. Chem., 1996, vol. 100, p. 13090.

3. Zangwill A. Physics at Surfaces. Cambridge, Cambridge University Press, 1990.

4. Ziff R.M., Gulari E., Barshad Y. // Phys. Rev. Lett., 1986, vol. 56, p. 2553.

5. Rettner C.T., Auerbach D.J., Tully J.C., Kleyn A.W. // J. Phys. Chem., 1996, vol. 100, p. 13021.

6. Zhdanov V.P. // Phys. Rev. Lett., 1996, vol. 77, p. 2109.

7. Hildebrand M., Mikhailov A.S., Ertl G. // Phys. Rev. E, 1998, vol. 58, p. 5483.

8. Grinstein G., Lai Z.-W., Browne D.A. // Phys. Rev. A, 1989, vol. 40, p. 4820.

9. Dickman R. // Phys. Rev. A, 1986, vol. 34, p. 4246. 
10. Luque J.J. // Phys. Rev. A, 1990, vol. 42, p. 3319.

11. Jensen I., Fogedby H.C. // Phys. Rev. A, 1990, vol. 42, p. 1969.

12. Luque J.J. // Phys. Rev. A, 1992, vol. 45, p. 7085.

13. Lemos M.C., Luque J.J., Jimenes-Morales F. // J. Chem. Phys., 1998, vol. 109, p. 8069.

14. Badiali J.P., Blum L., Rosinberg M.L. // Chem. Phys. Lett., 1986, vol. 129, p. 149.

15. Huckaby D.A., Blum L. // J. Chem. Phys., 1990, vol. 92, p. 2646.

16. Wertheim M.S. // J. Stat. Phys., 1984, vol. 35, p. 19; 1984, vol. 35, p. 35.

17. Wertheim M.S. // J. Chem. Phys., 1986, vol. 85, p. 2929.

18. Chang J., Sandler S.I. // J. Chem. Phys., 1995, vol. 102, p. 437.

19. Vakarin E.V., Duda Yu., Holovko M.F. // J. Stat. Phys., 1997, vol. 88, p. 1333;

Vakarin E.V., Duda Yu., Holovko M.F. // Mol. Phys., 1997, vol. 90, p. 611;

Vakarin E.V., Duda Yu., Holovko M.F. // J. Chem. Phys., 1997, vol. 107, p. 5569.

20. Holovko M.F., Vakarin E.V. // Mol. Phys., 1995, vol. 84, p. 1057.

21. Holovko M.F., Vakarin E.V. // Mol. Phys., 1996, vol. 87, p. 1375.

22. Voronov A., Luzinov I., Minko S., Sidorenko A., Vakarin E., Holovko M. // Macromolecules, 1997, vol. 30, p. 6929.

23. Kalyuzhnyi Yu.V., Protsykevytch I.A., Holovko M.F. // Chem. Phys. Lett., 1993, vol. 215 , p. 1.

24. Tammaro M., Evans J.W. // Phys. Rev. E, 1995, vol. 52, p. 2310.

25. Holovko M.F., Vakarin E.V. // Chem. Phys. Lett., 1994, vol. 230, p. 507.

26. Zhdanov V.P. // J. Chem. Phys., 1991, vol. 95, p. 2162.

27. Zaluska-Kotur M.A., Turski L.A. // Phys. Rev. B, 1994, vol. 50, p. 16102. 


\title{
Моделювання елементарних поверхневих процесів, що відбуваються в гетерогенних каталітичних реакціях
}

\author{
М.Ф.Головко, Е.В.Вакарін \\ Інститут фізики конденсованих систем НАН України, \\ 79011 Львів, вул. Свєнціцького, 1 \\ Отримано 18 квітня 2000 р.
}

Подано короткий огляд теоретичних результатів для схем реакцій Лангмюра-Гіншельвуда та Елі-Ріделя. Показано, що ступені елементарних процесів на поверхні є зв'язані через об'ємні властивості адсорбованих компонент. Це пов'язано залежністю між хімічним потенціалом асоціативно-дисоціативного балансу і густиною адсорбату біля поверхні. Показано, що адсорбція є чутливою до механізму димерної адсорбції (асоціативної або дисоціативної). Дисоціативна адсорбція веде до немонотонної залежності покриття від поверхневої активності. Одночасно ступінь дисоціації зростає з ростом адсорбції. 3 асоціативною адсорбцією пов'язана можливість існування в області малих густин критичної точки, в околі якої адсорбційні ізотерми не є неперервними. Це приводить до аномально низької поверхневої дифузії в області малих густин. Коротко обговорюється вплив цих результатів на кінетичні властивості.

Ключові слова: гетерогенний каталіз, двохмірний гратковий газ, дисоціативна та асоціативна адсорбція, поверхнева дифузія

PACS: $68.10 . J y, 82.65 . J V$ 